Bangladesh Journal of Neuroscience 2012; Vol. 28 (2) : 132-140

\title{
A Case of Idiopathic Chronic Inflammatory Demyelinating Polyradiculoneuropathy
}

\author{
MONIRUZZAMAN BHUIYAN ${ }^{1}$, MOHAMMAD NAJIM UDDIN ${ }^{2}$, MD MASUD RANA ${ }^{3}$, ANIS AHMED ${ }^{3}$, \\ MD RAFIQUL ISLAM ${ }^{4}$, HASAN ZAHIDUR RAHMAN $^{2}$, AK M ANWARULLAH $^{4}$
}

\begin{abstract}
:
A 40 years old man presented with progressive weakness and distal paraesthesia of limbs with difficulty in walking for last seven years. As the patient was not investigated thoroughly, a diagnostic dilemma was persisted. This patient went abroad \& investigated thoroughly and yet not reached to a confirm diagnosis. Subsequently this patient reported to us and diagnosed it to be a case of Idiopathic Chronic Inflammatory Demyelinating Polyradiculoneuropathy (CIDP) responded to oral steroid therapy. After follow-up, this patient showed marked improvement. So diagnostic dilemma that persisted with such a patient that showed improvement with treatment and a challenge to a treatable condition had encouraged us to report the case.
\end{abstract}

Keyword: Chronic Inflammatory Demyelinating Polyradiculoneuropathy, Idiopathic, Steroid.

\section{Introduction:}

Chronic inflammatory demyelinating polyradiculoneuropathy (CIDP) is a clinically heterogeneous, grossly symmetric, sensory and motor neuropathy evolving as a monophasic, relapsing, or progressive disorder. It develops over more than 8 weeks, distinguishing the condition from Guillain-Barre syndrome (GBS), which has an acute onset.

CIDP is thought to have an immune basis, its hallmark being inflammatory-mediated demyelination ${ }^{1}$. According to Burns ${ }^{2}$, Eichhorst described the first case of chronic and recurrent polyneuritis in 1890, and a few similar cases were reported during the following decades. During the 1950s, the concept of steroid-responsive chronic or relapsing polyneuritis emerged ${ }^{3}$. Along with experimental studies, this notion pointed to an immune mechanism. Later, investigators showed that reduced conduction velocities and conduction block resulted from segmental demyelination, which was considered typical findings of CIDP and related disorders ${ }^{4}$.For years, various terms were used to describe the condition and in 1982, Dyck and coworkers ${ }^{5}$ used the term chronic inflammatory demyelinating polyradiculoneuropathy, which summarizes its clinic pathological features.

Clinical trials have subsequently proven the efficacy of immunotherapy in patients with CIDP, and, to aid recognition of these patients, the American Academy of Neurology (AAN) proposed research diagnostic criteria ${ }^{6}$.During the following years these criteria seemed insufficiently sensitive for clinical practice, so new sets were proposed by several experts $^{7}$.In 2008, the French CIDP Study Group provided recommendations on diagnostic strategies for typical and atypical cases to help to improve diagnosis of this neuropathy ${ }^{8}$.

Although most patients with CIDP are recognized, since we still do not have definitive diagnostic tests, diagnosis can sometimes be challenging. Moreover, the prevalence of CIDP, which varies roughly between one and seven in 100000 across studies, is probably underestimated, partly because of an absence of recognition of possible or probable cases $^{9}$. In view of its clinical variability, the

1. Associate Professor, Department of Neurology, Bangabandhu Sheikh Mujib Medical University, Dhaka

2. MD Final part student, Department of Neurology, Bangabandhu Sheikh Mujib Medical University, Dhaka

3. Medical Officer, Department of Neurology, Bangabandhu Sheikh Mujib Medical University, Dhaka

4. Professor, Department of Neurology, Bangabandhu Sheikh Mujib Medical University, Dhaka 
diagnosis of CIDP should be envisaged during investigation of almost any multifocal or generalized neuropathy of unknown cause. This consideration is important because the condition is treatable ${ }^{10}$. Diagnosis is sometimes challenging and can require use of imaging and nerve biopsy. Raised protein concentrations in CSF and heterogeneous slowing of nerve conduction are typical of the condition. Steroids and intravenous immunoglobulin are effective, and plasma exchange can be helpful as rescue therapy. The usefulness of immunosuppressants needs to be established. The identification of specific diagnostic markers and new therapeutic strategies with conventional or targeted immunotherapy are needed to improve the outlook for patients with CIDP. Recent advances have been made in CIDP and other immunemediated neuropathies.

Herein, we present a case of CIDP-I in a middle aged man presented with progressive weakness, paresthesia \& numbness of all limbs with significant impairment of walking for last seven years and no systemic complaints and that was diagnosed with clinical findings and specific investigation and typed by literature review.

\section{Case Report}

A 40 years old Bangladeshi Bengali man admitted in Bangabandu Sheikh Mujib Medical University Hospital with complains of gradually increasing weakness of all the limbs for last 7 years. Initially weakness started in right hand and after few months the left hand was affected. Then the both lower limbs were affected. The weakness was gradually increasing as he had difficulty in holding objects and also had difficulty in walking. These symptoms were associated with wasting of the muscles. He also complaints of tingling and numbness of both hands and feet without bladder bowel involvement.

He was non-smoker, non-alcoholic, non-diabetic and non-hypertensive and hailing from non-arsenic prone area. He was married without promiscuity of any kind \& no one of his family member was suffering from similar disease. He had no occupational exposure to environmental toxin and on average Bangladeshi food. He had no joint pain or any systemic diseases.
Examination of the patient revealed that he was of average build with Pulse $80 / \mathrm{min}$. B.P 130/80 $\mathrm{mmHg} \&$ mild bilateral non tender lymphadenopathy of both axilla measuring $2 \mathrm{~cm}$ $\times 1.5 \mathrm{~cm}$ was found. He was not anemic and clubbing of the fingers, edema of legs and jaundice were absent and featureless abdomen without any organomegaly. Neurological examination revealed that higher psychic function was normal. Speech was normal. All the cranial nerves on both sides were normal. Motor system examination revealed wasting of muscles of hand \& feet on both side and tone was mildly reduced. Muscle power was Grade-4. All the deep tendon reflexes were diminished but the ankle jarks on the right side was absent.Babinski signs were absent There was foot drop on right side. His gait was stamping with foot drop on right side. Sensory function showed that there was $45 \%$ of hypoaesthesia and reduced pain sensation in the both hands and feet. Romberg's test was negative. No other neurological abnormality was found including no enlarged palpable nerve.

Investigation of the patient showed $\mathrm{Hb} \%$ was $80 \%$, ESR-75mm in 1st hour, differential count of WBC (N- 59\%, L-28\%,M-6\%,E-5\%, B-2\%) and morphology HCT-37.5\%, MCV-83.6fl, MCH-25.9pg, MCHC-310g/L and Platelet- 301×10/L. RBS$6.36 \mathrm{mmol} / \mathrm{L}$. Serum urea, creatinine \& electrolyte were within normal limit. Serum bilirubin -6. $6 \mu \mathrm{mol} /$ L, Total protiem-69. $8 \mathrm{~g} / \mathrm{L}, \mathrm{S}$. Albumin - 35gm/L, S. Globulin - 34. 8gm/L, AlK. Phosphatase-100unit/L, Alanine Aminotrasferase-32unit/L, Aspartate Aminotrasferase-14unit/L, $\gamma$-glutamyl trasferase- 67 unit/L, Lactate dehydrogenase- 146 unit/L, Creatinine kinase- 82unit/ L, P.T.-9.4 sec. APTT$24.8 \mathrm{sec}$. Routine urine \& stool examination was normal. Rh. Factor was negative. C. Reactive protein-10 mg/L. Double stranded DNA and Antinuclear antibody was negative. Serum c- ANCA was positive in 1:40 dilution. Serum HBsAg (-) negative. HIV antibody (-) negative. Serum protein electrophoresis showed Albumin -38.8\%, $\alpha$ globulin-17. 5\%, $\beta$-globulin-11.8,-globulin-24.9\%. S. Folate- $3.2 \mathrm{ng} / \mathrm{ml}$, Vit. B12-456pg $/ \mathrm{ml}$. S. Amylase36unit/L, S. Lead-0. 70 /L, S. Cu.-18. 6 mol / L, S. 
Zn.-12 mmol/L. FT3-5. $36 \mathrm{pmol} / \mathrm{L}$. FT4-23.13pmol/ L. TSH-2. $80 \mathrm{mIU} / \mathrm{L}$. S.Ca. $-2.23 \mathrm{mmol} / \mathrm{L}$. S. Inorganig phosphatase- $0.93 \mathrm{mmol} / \mathrm{L}$. S.Magnesium$0.79 \mathrm{mmol} / \mathrm{L}$. USG of abdomen was normal. C.T. Scan of brain was normal. Abdominal aortogram \& selective bilateral renal angiography showed no sign of vascularity or microaneurysm was found. Histopathology of left axillary lymph node showed reactive changes without evidence of malignancy \& Reed-Sternberg cells. Bone marrow examination was normal and storage of iron is normal. CSF was clear, CSF protein $65 \mathrm{mg} / \mathrm{dl}$, CSF WBC- nil,
RBC- $80 / \mathrm{mm}^{3}$ \& Gram Staining showed no organism \& C/S was negative. CSF culture for AFB \& virus was negative and both CSF \& blood electrophoresis revealed no oligoclonal band. Right Sural nerve biopsy showed several nerve bundles along with several medium sized blood vessels in the epineurium. There is no evidence of interstitial or perivascular inflammatory infiltrates or abnormal deposits. Special stain for myelin showed moderate to severe loss of myelinated fibers with relative axonal preservation. Findings NCS of crossed limbs showed in Table -1

Table-I

Motor nerve conduction

\begin{tabular}{lccccccc}
\hline $\begin{array}{l}\text { Median nerve, Right } \\
\text { Stimulus site }\end{array}$ & $\begin{array}{c}\text { Lat 1 } \\
(\mathrm{ms})\end{array}$ & $\begin{array}{c}\text { Lat 2 } \\
(\mathrm{ms})\end{array}$ & $\begin{array}{c}\text { Fwave } \\
\text { latency }(\mathrm{ms})\end{array}$ & $\begin{array}{c}\text { Duration } \\
(\mathrm{ms})\end{array}$ & Amp (mV) & Area & NCV (m/s) \\
\hline Wrist & 2.8 & 7 & 4.1 & 4.2 & 1.4 & 3.2 & 48 \\
Elbow & 7.8 & 11.7 & & 3.9 & 1.2 & 2.5 & \\
Ulnar nerve, Right & & & & & & & \\
Wrist & 2.5 & 7.2 & 2.6 & 4.7 & 1 & 2.8 & 55.3 \\
Elbow & 7.2 & 13.4 & & 6.2 & 1.2 & 3.6 & \\
Peroneal nerve, Left & $\mathrm{Amp}(\mu \mathrm{V})$ & & & & & & \\
Ankle & 3.9 & 8 & & 4.1 & 90 & 208 & 40.5 \\
Fibular head & 11.8 & 16.6 & & 4.8 & 151 & 444 & \\
\hline
\end{tabular}
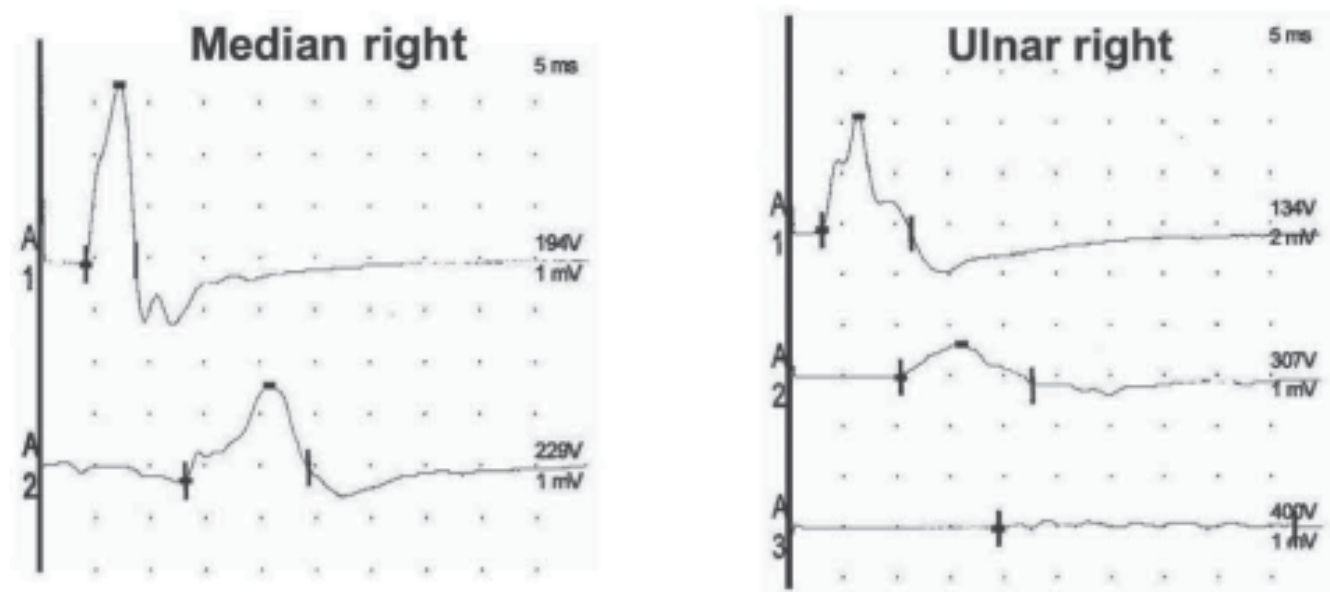

Fig.-1: Nerve conduction study before treatment illustrating a conduction block on the right ulnar and temporal dispersion on the median nerves. 
Electrophysiological findings were suggestive of chronic inflammatory demyelinating polyradiculoneuropathy (Table 1 \& Fig. 1).

Patient was treated with oral prednisolone $60 \mathrm{mg}$ once a day showed moderate improvement after one month as he can hold objects and walk without assistance and also there was improvement of sensory symptoms. Patient was be followed-up in neurology outpatients department after 3 months and electrophysiological study was carried out which showed improvement from previous study \& slow tapering of steroid was done subsequently.

\section{Discussion:}

CIDP is relatively uncommon, with prevalence between one in 100000 in southeast UK ${ }^{11}$ and 7.7 in 100000 in northern Norway ${ }^{12}$. In all studies, CIDP was observed to be most frequent in adult men, as was reported in a Japanese population with an annual incidence of 0.48 per $100000^{13}$. CIDP is regarded as an autoimmune disease involving cellular and humoral immunity. However, by contrast with GBS, a single triggering antigen has not yet been found, except in rare cases of CIDP associated with melanoma, in which tumour cells share carbohydrate epitopes with Schwann cells ${ }^{14}$.

After the first description by Dyck and co-workers ${ }^{1}$, the clinical pattern of CIDP was defined by several features: (i) selective involvement of the peripheral nervous system; (ii) involvement of proximal as well as distal limb structures; (iii) involvement of both motor and sensory fibers (although in some cases motor or sensory fibers only might be affected); and (iv) a recurrent, continuously worsening, or fluctuating course. The classic pattern of limb involvement in CIDP is then a sensory and motor diffuse polyneuropathy with generalized areflexia, and proximal involvement evolving over more than 2 months ${ }^{6}$. Cranial nerves are occasionally affected, with a particular tropism for the VIIth pair, but ophthalmoplegia or bulbar weakness can be present ${ }^{15}$. The disease course is usually separated into monophasic, progressive, and relapsing forms, although a strict definition of the relapsing form has seldom been used in the literature.
Several variants have been described on the basis of distribution of symptoms and signs16.Although controversies have emerged as to whether some of these syndromes are distinct clinical entities $^{17}$. The common pathogenic mechanism of inflammatory demyelination clearly shows that they belong to the spectrum of $\mathrm{CIDP}^{18}$. Moreover, identification of patients with these variants is crucial because they respond to immunomodulatory therapy as well as patients with the classic phenotype.

CIDP can be associated with various conditions, including hepatitis $\mathrm{C}$, inflammatory bowel disease, and Iymphoma, monoclonal gammopathy of undetermined significance (MGUS), HIVIAIDS infection, organ transplant, and connective tissue disorders. Similarly, investigators have reported that CIDP was more frequent in patients with diabetes mellitus than it was in the general population ${ }^{19}$, although this assumption has been challenged ${ }^{20}$. Several reports have shown subclinical involvement of the central pathways in some patients, suggesting that CIDP might involve the central as well as the peripheral nervous system $^{21}$. This finding seems to differ from that of a population of patients in whom there is an association between a CIDP-like disorder and a multiple sclerosis-like condition, in which clinical involvement of the peripheral as well as the central nervous system is obvious ${ }^{22}$. Finally, CIDP has been reported in patients with Charcot-Marie-Tooth disease ${ }^{23}$, suggesting that superimposed inflammatory mechanisms might occur in hereditary neuropathies and contribute to disability ${ }^{24}$.

Childhood CIDP has been extensively reported, allowing a precise picture to be drawn of the presentation, response to treatment, and prognosis ${ }^{25}$. Overall, children with CIDP have a more rapid onset, greater disability at the peak of the disease, and a more frequent relapsing course than do adults, but respond better to treatment and have a more favourable long-term outcome.

Nerve conduction findings are a key part of diagnostic investigation for patients with suspected CIDP. Indeed, slowing of nerve conduction mainly occurs during nerve demyelination, either diffuse or segmental, and is highly suggestive of CIDP in 
the appropriate clinical situation ${ }^{1}$. Following the work of Albers and Kelly, 85 consensus electrophysiological research criteria for CIDP were elaborated in the early $1990 \mathrm{~s}^{6}$. Several variables were considered: (i) slowing of motor nerve conduction velocities; (ii) lengthening of distal motor latencies; (iii) prolonged minimal F wave latencies; and (iv) partial conduction block. The severity of slowing in individual nerves and the number of nerves in which abnormalities had to be found were carefully chosen to be specific enough for research purposes, but several investigators have judged the sensitivity of these criteria to be insufficient for clinical practice.Therefore, several sets of electrophysiological criteria for CIDP have been proposed to improve detection of patients with this treatable disorder ${ }^{7}$. Thaisetthawatkul and colleagues ${ }^{26}$ showed that dispersion of distal motor action potentials was another useful criterion, and others, including a panel of experts from the European Federation of Neurological Societies (EFNS) and the Peripheral Nerve Society (PNS), have proposed that this measure be included in electrophysiological criteria for CIDP ${ }^{27}$. The EFNS/ PNS criteria seem to provide the best chance of identification of patients with CIDP and whether axonal forms of CIDP exist has been a matter of debate. Axonal involvement in this primary demyelinating neuropathy is obvious in many cases and is closely linked to disability. Uncini and coworkers ${ }^{28}$ described five patients with a condition they called steroid-responsive axonal polyneuropathy, suggesting that it was an axonal variant of CIDP. However, whether the inflammatory process directly involves axons, the condition being the chronic counterpart of acute motor sensory axonal neuropathy, is far from proven from this study. Indeed, patients who do not meet neurophysiological criteria for a primary demyelinating neuropathy might have evidence of inflammatory demyelination on nerve biopsy².

Many patients with CIDP have raised CSF protein with leukocyte counts lower than 10 WBC per ìL, which is indicative of root inflammation, and this disease criterion was in the past regarded as mandatory $^{1}$. However, as has long been recognized, protein content can be normal in at least $10 \%$ of patients, and the criterion is now considered supportive ${ }^{6}$. When leukocyte count is raised in the CSF, Lyme borreliosis, HIVIAIDS infection, lymphoma, or sarcoidosis should be considered. Since root involvement is not accessible to conventional nerve conduction studies, somatosensory evoked potentials have been investigated in patients with chronic demyelinating neuropathies ${ }^{29}$. This technique might be especially useful when assessing for proximal involvement of sensory nerves in patients with normal sural sensory potentials. Similarly, breakdown of the blood-nerve barrier at the root level could cause contrast enhancement on $\mathrm{MRI}^{30}$.Moreover, root hypertrophy can be shown by MRI at the lumbar or cervical level in patients with CIDP and can occasionally cause clinical findings that are attributable to lumbar or cervical stenosis $^{30}$. Because inflammation can be widespread along nerves in CIDP, contrast enhancement and hypertrophy are sometimes shown by conventional MRI at the plexus level ${ }^{31}$. Besides conventional MRI sequences, diffusion neurography, which is a modified diffusionweighted MRI technique, might be a promising method to identify abnormalities in nerves of patients with CIDP.CIDP mainly affects large myelinated fibers, small fibers are not unaffected, as shown by nerve and skin biopsy findings ${ }^{32}$. The chronic proximal demyelinating lesions frequently induce distal axon degeneration with dropout of large myelinated fibers. On paraffin sections, mononuclear cell infiltrates can be seen as scattered cells, often surrounding vessels. These infiltrates can be seen in the endoneurium, as well as in the perineurium and the epineurium by optic microscopy, but are sometimes more clearly seen by ultrastructural examination. However, the presence of these small clusters of inflammatory cells is uncommon in nerve biopsy samples from patients with CIDP and is not mandatory for the diagnosis. Demyelination and remyelination are pathological hallmarks of CIDP. Segmental demyelination is best seen on teased fiber preparations. The value of nerve biopsy in diagnosis of patients with supposed CIDP has been debated. However, most agree that biopsy should be done 
in patients with atypical clinical or neurophysiological findings, and could be considered in patients with poor response to treatment to rule out alternative diagnoses.

CIDP can be associated with some general disorders (paraproteinaemia, diabetes, thyroid dysfunction, neoplasm), probably as a result of a dysimmune process ${ }^{33}$, but the association with MGUS is the most common. Indeed, some patients with CIDP have a concomitant IgG MGUS. There is no evidence from published work and our personal experience that these patients should be considered and treated differently from those without monoclonal gammopathy ${ }^{33}$. In some cases of malignant gammopathies, a direct link to the neuropathy needs to be eliminated by nerve biopsy because the monoclonal component or malignant cells can sometimes infiltrate the nervous parenchyma. If the monoclonal paraprotein is an $\lg \mathrm{M}$, the distinction between an anti-myelin associated glycoprotein (MAG) neuropathy and CIDP is based on an anti-MAG assay. Most patients with anti-MAG neuropathy are of middle or old age and have a pure sensory ataxic or painful neuropathy, with nerve conduction studies usually showing a disproportionate lengthening of terminal latencies and a severe decrease in motor and sensory responses in the lower limbs.Although CIDP and anti-MAG neuropathy are regarded as distinct entities, there might be some overlap at the clinical, neurophysiological, and neuropathological levels; response to immunotherapy, however, is usually poor in patients with anti-MAG neuropathy. Diagnosis of the multifocal form of CIDP can be challenging, and misdiagnosis with multiple entrapment neuropathy or multifocal motor neuropathy with conduction block is common because of the frequent upper-limb predominance of the three conditions. Similarly, conduction block because of partial nerve ischaemia might erroneously suggest segmental demyelination in patients with multiple mononeuropathy due to necrotizing vasculitis ${ }^{34}$.Another challenging issue is the polyneuropathy of POEMS syndrome (polyneuropathy, organomegaly, endocrinopathy, $M$ protein, skin changes), which is very similar to CIDP $^{34}$. It does not usually respond to conventional immunotherapy, but rather necessitates radiation therapy for solitary lesions or high-dose chemotherapy.Since the polyneuropathy of POEMS syndrome is usually rapidly disabling, its diagnosis can be a matter of urgency. Nerve biopsy can show specific lesions ${ }^{36}$, but the presence of a lambda light chain along with sclerotic bone lesions is usually sufficient to make the diagnosis. Moreover, high serum concentrations of vascular endothelial growth factor are a very useful and specific diagnostic measure for this condition ${ }^{36}$. At any age, but chiefly if patients have a predominantly distal weakness or poor response to treatment, a hereditary neuropathy such as Refsum's disease, Charcot-Marie- Tooth disease, or transthyretin amyloid neuropathy ${ }^{37}$ should be ruled out. Finally, the first diagnosis in some patients with CIDP will be chronic idiopathic axonal neuropathy or cryptogenic sensory polyneuropathy, and the disease will be allowed to progress without intervention despite the availability of effective therapy. After some time, any chronic demyelinating neuropathy will induce axonal loss. Patients who do not meet electrodiagnostic criteria for CIDP might benefit from extensive tests including nerve biopsy to look for possible CIDP, especially young patients and those with an aggressive course or prominent motor involvement, since they could benefit from immunotherapy².

Steroids have been widely used in CIDP since a single randomized controlled trial showed the efficacy of prednisone ${ }^{6}$. Since then, the usefulness of steroids in CIDP has been confirmed and a controlled study showed that a 6-week course of $60 \mathrm{mg}$ daily oral prednisolone with rapid tapering is as effective as one course of IVIg at $2 \mathrm{~g} / \mathrm{kg}^{7}$. Therefore, recent consensus guidelines concluded that "a trial of steroids should be considered in all patients with CIDP and significant disability"9.Continuous oral steroid therapy is the commonest regimen used, but some have proposed pulsed high-dose treatment with dexamethasone ${ }^{45}$ or oral methylprednisolone ${ }^{46}$.Steroid treatment seems to be beneficial in $60-70 \%$ of patients with CIDP $^{47}$, and long-term treatment needs to be individually tailored according to disease course, with careful attention paid to potential side-effects. 
Choice of treatment will depend on several variables, and in particular initial disease severity, age, general health status, and potential contraindications to steroids or IVIg Around $15-30 \%$ of patients will not relapse after this first course ${ }^{8}$, and for patients who need additional treatment, dose and intervals between courses should be individually tailored to achieve the most cost-effective regimen. If a patient does not respond to one of these first-line therapies, switching to the other is advisable. PE or a combination of steroids and IVIg can be started if neither of these treatments proves effective. Refractory cases might need intensive immunosuppression, as has been proposed by some investigators ${ }^{47}$. Long-term maintenance therapy will require careful attention because of side-effects of treatments on the one hand, and because of the risk of relapse and axonal loss on the other.

Available data suggest that the long-term prognosis of CIDP is highly dependent on age at onset, clinical form of the disease, and initial response to treatment. Young patients with a rapid (subacute) onset or a monophasic course are more likely to respond to treatment and recover completely ${ }^{48}$. The effect of age of onset seems especially important in elderly people, for whom full recovery after treatment is less frequent than for juvenile patients or adults aged younger than 64 years ${ }^{49}$. Among the clinical variables, proximal weakness has been linked to a higher remission rate and better prognosis compared with the distal phenotype ${ }^{48}$. Overall, studies suggest that long-term outcome is usually good in patients with CIDP, especially in those with a monophasic or relapsing course $^{48}$.

\section{Conclusions:}

CIDP is a potentially disabling immune-mediated neuropathy. Its diagnosis is important because the condition is responsive to several disease-modifying therapies such as steroids, IVIg, PE, and immunosuppressants. In view of recent studies suggesting that prevalence of CIDP has been largely underestimated, we think investigation in a patient suspected of having CIDP should use all available methods, including nerve biopsy. The strategy for diagnosis of CIDP has been reviewed elsewhere ${ }^{12}$ and should chiefly be based on clinical presentation and electrodiagnostic evaluation. Ultimately, a trial of steroids or IVIg seems reasonable if sufficient evidence suggests the diagnosis is at least probable. Future research is needed to identify disease markers to improve diagnosis and to develop new therapeutic strategies based on available and emerging therapies.

\section{Conflict of Interest: None}

\section{References:}

1. Dyck PJ, Lais AC, Ohta M, Bastron JA, Okazaki H, Groover RV. Chronic inflammatory polyradiculoneuropathy. Mayo Clin Proc 1975; 50: 621-37.

2. Burns TM. Chronic inflammatory demyelinating polyradiculoneuropathy. Arch Neurol 2004; 61: 973-75.

3. Austin JH. Recurrent polyneuropathies and their corticosteroid treatment. Brain 1958; 81: 11-192.

4. Lewis RA, Sumner AJ. The electrodiagnostic distinctions between chronic familial and acquired demyelinative neuropathies. Neurology 1982; 32: 592-96.

5. Dyck PJ, O'Brien PC, Oviatt KF, Dinapoli RP, Daube JR, Bartleson JD, Mokri B, Swift T, Low PA, WindebankAJ. Prednisone improves chronic inflammatory demyelinating polyradiculoneuropathy more than no treatment. Ann Neurol 1982; 11: 136-41.

6. American Academy of Neurology AIDS Task Force. Research criteria for diagnosis of chronic inflammatory demyelinating polyneuropathy (CIDP). Neurology 1991; 41: 617-18.

7. Hughes $\mathrm{R}$, Bensa $\mathrm{S}$, Willison $\mathrm{H}$, Van den Bergh P, Comi G, Illa I, Nobile-Orazio E, van Doorn P, Dalakas M, Bojar M, Swan A. Randomized controlled trial of intravenous immunoglobulin versus oral prednisolone in chronic inflammatory demyelinating polyradiculo neuropathy. Ann Neurol 2001; 50: 195-201. 
8. French CIDP Study Group. Recommendations on diagnostic strategies for chronic inflammatory demyelinating polyradiculoneuropathy. J Neurol Neurosurg Psychiatry 2008; 79: 115-18.

9. Rajabally YA, Simpson BS, Beri S, Bankart J, Gosalakkal JA.Epidemiologic variability of chronic inflammatory demyelinating polyneuropathy with different diagnostic criteria: study of a UK population. Muscle Nerve 2009; 39: 432-38.

10. Magy L, Vallat JM. Evidence-based treatment of chronic immune-mediated neuropathies. Expert Opin Pharmacother 2009; 10: 1741-54.

11. Lunn MP, Manji H, Choudhary PP, Hughes RA, Thomas PK.Chronic inflammatory demyelinating polyradiculoneuropathy: a prevalence study in south east England. J Neurol Neurosurg Psychiatry 1999; 66: 677-80.

12. Mygland A, Monstad P. Chronic polyneuropathies in Vest-Agder, Norway. Eur J Neurol 2001; 8: 157-65.

13. M lijima,H Koike, $N$ Hattori,A Tamakoshi, $M$ Katsuno,F Tanaka,M Yamamoto,K Arimura,G Sobue.Prevalence and incidence rates of chronic inflammatory demyelinating polyneuropathy in the Japanese population. J Neurol Neurosurg Psychiatry 2008; 79: 1040-43.

14. Koller H, Schroeter M, Kieseier BC, Hartung HP. Chronic inflammatory demyelinating polyneuropathy-update on pathogenesis, diagnostic criteria and therapy. Curr Opin Neurol 2005; 18: 273-78.

15. Gorson KC, Allam G, Ropper AH. Chronic infl amatory demyelinating polyneuropathy: clinical features and response to treatment in 67 consecutive patients with and without a monoclonal gammopathy. Neurology 1997; 48: 321-28.

16. Busby M, Donaghy M. Chronic dysimmune neuropathy. A subclassification based upon the clinical features of 102 patients. J Neurol 2003; 250: 714-24.

17. Katz JS, Saperstein DS, Gronseth G, Amato AA, Barohn RJ. Distal acquired demyelinating symmetric neuropathy. Neurology 2000; 54 : 615-20.
18. Oh SJ, LaGanke C, Powers R, Wolfe GI, Quinton RA, Burns DK. Multifocal motor sensory demyelinating neuropathy: inflammatory demyelinating polyradiculoneuropathy. Neurology 2005; 65: 1639-42.

19. Stewart JD, McKelvey R, Durcan L, Carpenter S, Karpati G. Chronic inflammatory demyelinating polyneuropathy (CIDP) in diabetics. J Neurol Sci 1996; 142: 59-64.

20. Hawke SH, Hallinan JM, McLeod JG. Cranial magnetic resonance imaging in chronic demyelinating polyneuropathy. J Neurol Neurosurg Psychiatry 1990; 53: 794-96.

21. Mendell JR, Kolkin S, Kissel JT, Weiss KL, Chakeres DW, Rammohan KW. Evidence for central nervous system demyelination in chronic inflammatory demyelinating polyradiculoneuropathy. Neurology 1987; 37: 1291-94.

22. Sharma KR, Saadia D, Facca AG, Bhatia R, Ayyar DR, Sheremata W. Chronic inflammatory demyelinating polyradiculoneuropathy associated with multiple sclerosis. J Clin Neuromuscul Dis 2008; 9: 385-96.

23. Ginsberg L, Malik O, Kenton AR, Sharp D, Muddle JR, Davis MB, Winer JB, Orrell RW, King $\mathrm{RH}$. Coexistent hereditary and inflammatory neuropathy. Brain 2004; 127: 193-202.

24. Dyck PJ, Swanson CJ, Low PA, Bartleson JD, Lambert EH. Prednisone-responsive hereditary motor and sensory neuropathy. Mayo Clin Proc 1982; 57: 239-46.

25. Nevo Y, Pestronk A, Kornberg AJ, Connolly AM, Yee WC, Iqbal I, Shield LK. Childhood chronic inflammatory demyelinating neuropathies: clinical course and long-term follow-up. Neurology 1996; 47: 98-102.

26. Thaisetthawatkul P, Logigian EL, Herrmann DN. Dispersion of the distal compound muscle action potential as a diagnostic criterion for chronic inflammatory demyelinating polyneuropathy. Neurology 2002; 59: 1526-32.

27. European Federation of Neurological Societies/Peripheral Nerve Society Guideline on management of chronic inflammatory 
demyelinating polyradiculoneuropathy. Report of a joint task force of the European Federation of Neurological Societies and the Peripheral Nerve Society. J Peripher Nerv Syst 2005; 10: 220-28.

28. Uncini A, Sabatelli M, Mignogna T, Lugaresi A, Liguori R, Montagna P. Chronic progressive steroid responsive axonal polyneuropathy: a CIDP variant or a primary axonal disorder Muscle Nerve 1996; 19: 365-71.

29. Yiannikas C, Vucic S. Utility of somatosensory evoked potentials in chronic acquired demyelinating neuropathy. Muscle Nerve 2008; 38: 1447-54.

30. Duggins AJ, McLeod JG, Pollard JD, Davies L, Yang F, Thompson EO, Soper JR. Spinal root and plexus hypertrophy in chronic inflammatory demyelinating polyneuropathy. Brain 1999; 122: 1383-90.

31. Bradley LJ, Wilhelm T, King RH, Ginsberg L, Orrell RW.Brachial plexus hypertrophy in chronic inflammatory demyelinating polyradiculoneuropathy. Neuromuscul Disord 2006; 16: 126-31.

32. Chiang $M C$, Lin $\mathrm{YH}, \mathrm{Pan} \mathrm{CL}$, Tseng $\mathrm{TJ}$, Lin WM, Hsieh ST. Cutaneous innervation in chronic inflammatory demyelinating polyneuropathy. Neurology 2002; 59: 109498.

33. Vallat JM, Jauberteau MO, Bordessoule D, Yardin C, Preux PM, Couratier P. Link between peripheral neuropathy and monoclonal dysglobulinemia: a study of 66 cases. J Neurol Sci 1996; 137: 124-30.

34. Ropert A, Metral S. Conduction block in neuropathies with necrotizing vasculitis. Muscle Nerve 1990; 13: 102-05.

35. Kelly JJ Jr, Kyle RA, Miles JM, Dyck PJ. Osteosclerotic myeloma and peripheral neuropathy. Neurology 1983; 33: 202-10.

36. Vital C, Gherardi R, Vital A, Kopp N, Pellissier JF, Soubrier M, Clavelou P, Bellance R, Delisle MB, Ruchoux MM. Uncompacted myelin lamellae in polyneuropathy, organomegaly, endocrinopathy, M-protein and skin changes syndrome. Ultrastructural study of peripheral nerve biopsy from 22 patients. Acta Neuropathol 1994; 87: 302-07.
37. Plante-Bordeneuve V, Ferreira A, Lalu T. Diagnostic pitfalls in sporadic transthyretin familial amyloid polyneuropathy (TTR-FAP). Neurology 2007; 69: 693-98.

38. Molenaar DS, van Doorn PA, Vermeulen M. Pulsed high dose dexamethasone treatment in chronic inflammatory demyelinating polyneuropathy: a pilot study. J Neurol Neurosurg Psychiatry 1997; 62: 388-90.

39. Muley SA, Kelkar P, Parry GJ. Treatment of chronic inflammatory demyelinating polyneuropathy with pulsed oral steroids.Arch Neurol 2008; 65: 1460-64.

40. Nobile-Orazio E. Treatment of dysimmune neuropathies. J Neurol 2005; 252: 38595.116

41. Brannagan TH 3rd, Pradhan A, HeimanPatterson T.High-dose cyclophosphamide without stem-cell rescue for refractory CIDP. Neurology 2002; 58: 1856-58.146

42. Mygland A, Monstad P, Vedeler C. Onset and course of chronic inflammatory demyelinating polyneuropathy. Muscle Nerve 2005; 31: 58993.

43. Kuwabara S, Misawa S, Mori M, Tamura N, Kubota M, Hattori T.Long term prognosis of chronic inflammatory demyelinating polyneuropathy: a five year follow up of 38 cases. J Neurol Neurosurg Psychiatry 2006; 77: 66-70.

44. Hattori $\mathrm{N}$, Misu $\mathrm{K}$, Koike $\mathrm{H}$, Ichimura $\mathrm{M}$, Nagamatsu M, Hirayama M, Sobue G. Age of onset influences clinical features of chronic inflammatorydemyelinating polyneuropathy.J Neurol Sci 2001; 184: 57-63.

45. Mygland A, Monstad P, Vedeler C. Onset and course of chronic inflammatory demyelinating polyneuropathy. Muscle Nerve 2005; 31: 589-93.

46. Molenaar DS, van Doorn PA, Vermeulen M. Pulsed high dose dexamethasone treatment in chronic inflammatory demyelinating polyneuropathy: a pilot study. J Neurol Neurosurg Psychiatry 1997; 62: 388-90.

47. Muley SA, Kelkar P, Parry GJ. Treatment of chronic inflammatory demyelinating polyneuropathy with pulsed oral steroids. Arch Neurol 2008; 65: 1460-64.

48. Nobile-Orazio E. Treatment of dysimmune neuropathies. J Neurol 2005; 252: 385-95. 